\title{
/BIOQ\&A
}

\section{Fermentation and Cell Culture}

\author{
C OMPILED ANDEDITED \\ BY JOHN HODGSON
}

\author{
This month's \\ BioQ\&A looks \\ at issues and \\ problems in \\ fermentation \\ and cell \\ culture. The \\ answers come \\ from Berni \\ Roberts at \\ Chemap \\ (Volketswil, \\ Switzerland), \\ Nelson \\ Nazareth of Life \\ Science \\ Laboratories \\ (Luton, U.K.), \\ and Nick \\ Vosper of New \\ Brunswick \\ Scientific \\ (Edison, $N J$ ).
}

\begin{abstract}
Q. Antifoam does not affect the productivity of our system or its downstream processing. However, the addition of the antifoam agent does not control the foam as rapidly as we require. What can we do to improve this?
\end{abstract}

A. In general, the problem of foam can be tackled both by prevention and by treatment. One way of minimizing foam is to "cascade" the process parameters which influence foam generation. For instance, you could reduce gas-flow and agitation in the event of a foam buildup. Where foam is unavoidable, it is best to treat it as early as possible after formationwhile it is still nascent. This demands a good foam detection system. Timed dosing with antifoam is not always so effective and may lead to excessive antifoam additions. Finally, all antifoams are not equal: selecting the right one for your process is very important. (N.V.)

Q. Can my existing fermentor be used to culture insect or plant cells?

A. Eukaryote cells can be cultures in modified microbial fermentors, but the compromises necessary to reconcile oxygen transfer rate, foam generation and shear stress may cause suboptimal growth and gene expression. For optimal growth, you should use a system designed specially for eukaryote cells. (N.V.)

A. In general, benchtop, autoclavable glass vessels that are waterjacketed and have a hemispherical bottom can be used as dual-purpose reactors. The high-speed Rushton turbines and gas-sparging used for prokaryote culture can be replaced with marine impeller and gentle aeration for eukaryotes. Larger, in-situ-sterilizable vessels are usually not so versatile: however, while specifically designed "eukaryote" bioreactors have been used to grow prokaryotes efficiently, the reverse is less common. (N.N.)

Q. What is the best surface finish for cleanability and sterilization in fermentation vessels and process lines?

A. Appearances can be deceptive. The mirrorpolished finishes offered by many manufacturers have been adopted from the dairy industry where the requirements for cleaning and asepsis are totally different from those in the biopharmaceutical area. One of the cheapest finishes - pickled/electropolished-has been shown to be one of the best surfaces for cleanability and sterilizing. However, it has an unsatisfactory appearance and has not gained market acceptance. In practice, maintaining the surface is less dependent on the finish than on the cleaning regime used after the process: if not performed correctly, cleaning can render even the best surface useless. (B.R.)

Q. Do I really need to use double sealing systems to ensure containment of recombinant or pathogenic organism?

A. All the government agency guidelines recommend the use of double "O"-ring or elastomer seals on process vessels as a primary containment for biological processes. There are also secondary and tertiary containment measures such as building, and room design and the control of personnel, goods and products flows. In short, containment goes beyond the tank. (N.V.)

Q. Are magnetic couplings better than double mechanical seals for the culture agitation system?

A. Magnetic couplings eliminate the need for rotating seals, an advantage for containment. However, the magnetic assembly is bulkier than the mechanical equivalent and the bulk increases with scale. The tendency for the magnet to "lose" the agitator also increases with scale. In cell culture, where power requirements are relatively low, magnetic couplings are popular. However, some designs cause "grinding" of microcarriers or cells. (B.R.)

Q. Which of the many technologies for cell culture can be readily scale up to production levels?

A. Be wary of gimmicks and unusual devices for any process that is going into production. The best advice is to keep things simple for ease of scale up and to ensure that you don't get trapped in a technology which is not available at a larger scale. Many companies find that it is better to work backwards from what is possible at the large scale — to "scale down." (B.R.)

Bio/Technology invites readers and suppliers to contribute to the July BioQ\&A topic "Filtration." In June, BioQ\&A will answer general reader questions. Send all BioQ\&A correspondence to:

John Hodgson, Articles Editor, Bio/Technology, 4 Little Essex Street, London WC2R 3LF U.K. Fax: +44712402408. 